\title{
25 Evaluation im Projekt optes
}

\author{
Christian Schmidt, Victoria Marquardt, Gabi Reinmann, Daria Paul
}

\subsection{Theoretische Fundierung}

Von Beginn an wurde im optes-Verbundprojekt viel Wert darauf gelegt, dass die Projektarbeit gezielt durch Evaluationen begleitet wird. Der Nutzen dieser Maßnahmen liegt in einem fortlaufenden Prozess der (didaktischen) Qualitätssicherung (Reinmann 2004) sowohl der Ergebnisse des Projekts als auch der Prozesse, um diese Ergebnisse zu erzielen. Die Prozessevaluation diente zunächst dazu, die $\mathrm{Zu}$ sammenarbeit und den Austausch zwischen den Teilprojekten sukzessive zu verbessern; dies hatte auch indirekt Einfluss auf die Projektergebnisse. Sowohl Prozess- als auch Ergebnisevaluationen waren von Anfang an nicht nur summativ als Erfolgsmessung am Projektende angelegt, sondern fanden auch bereits formativ während der Projektlaufzeit (Scriven 1991) statt, um mit Erkenntnissen über Zwischenstände zyklische Verbesserungen (Re-Designs) vornehmen zu können. Die Evaluation im Rahmen des optes-Projekts lässt sich als praktisch-orientierte Evaluationsforschung verstehen (Wottawa 2006). Dies bedeutet, dass Evaluationen zwar mit wissenschaftlichen Methoden und nach wissenschaftlichen Standards durchgeführt wurden, das Erkenntnisinteresse jedoch immer darauf lag, (Teil-)Projektergebnisse und Arbeitsprozesse zu optimieren und die dazu erforderlichen Evaluationsmaßnahmen möglichst ressourcenschonend zu gestalten (Patton 2010). Die Evaluation im Rahmen von optes war somit stets ein Teil der didaktischen Qualitätssicherung (siehe Kapitel 24).

\subsection{Entwicklung der Evaluationsansätze im Projektverlauf - Von der ,Hilfe zur Selbsthilfe ${ }^{66}$ zum modularisierten Online-Fragebogen}

Mit fortschreitendem Projektverlauf haben sich die Ansätze zur Evaluation weiterentwickelt, wurden turnusmäßig überprüft und bei Bedarf an den jeweiligen Projektstand angepasst. Zu Beginn des optes-Verbundprojekts sind in den einzelnen Teilprojekten zunächst die geplanten Maßnahmen erarbeitet worden. In dieser Phase haben sich Evaluationsvorhaben auf die Zusammenarbeit im Projekt und auf Teilprojektergebnisse konzentriert. Die wissenschaftliche Begleitung hat hier vor allem die Koordinationsfunktion übernommen und die Partner bei der Planung und Erstellung von Erhebungsinstrumenten sowie bei der Auswertung und Dokumentation unterstützt, also „Hilfe zur Selbsthilfe“ geleistet. Dazu wurden Leitfäden mit

Die Originalversion dieses Kapitels wurde revidiert. Ein Erratum ist verfügbar unter https://doi.org/10.1007/978-3-658-31279-4_32 
grundlegenden Hinweisen zur Durchführung von Evaluationsmaßnahmen erstellt; zugleich erhielten die Partner individuelles Feedback zum Evaluationsprozess. So wurde gewährleistet, dass die Erhebungen einerseits zu den Erfordernissen an den unterschiedlichen Standorten sowie den Teilprojektspezifika passten, andererseits aber auch allgemeine Evaluationsstandards eingehalten wurden.

Die Evaluationsergebnisse haben die Projektpartner als Kurzberichte aufbereitet, sodass alle einen einheitlichen Informationsstand hatten. Die Kurzberichte wurden zudem von der wissenschaftlichen Begleitung analysiert, um auf diesem Wege erste Einschätzungen zur Projektwirkung vorzunehmen. Ergänzend dazu wurden die Durchführenden zu selbst beobachteten Wirkungen sowie eigenen Bewertungen und Begründungen befragt.

Mit dem Fortschreiten des Projekts ließen sich Teilprojektergebnisse zunehmend verknüpfen; in der Folge haben wir auch den Evaluationsansatz angepasst. In den Vordergrund rückte nun die Wirkungsevaluation der mit den optes-Materialien erstellten Lehr-Lern-Szenarien an den jeweiligen Partnerhochschulen. Hierzu wurde ein modularisierter Fragebogen erarbeitet, um einerseits den lokalen Besonderheiten an den jeweiligen Partnerhochschulen Rechnung zu tragen und anderseits eine gewisse Vergleichbarkeit der Daten zu gewährleisten. Auf diese Weise war es möglich, über verschiedene Standorte und Teilprojekte hinweg, Nutzerevaluationen durchzuführen, dabei größere Stichproben zu generieren, aussagekräftigere Datensätze zu erhalten und letztlich Best-Practice-Umsetzungen zu identifizieren. Dieser modularisierte Fragebogen wird bis heute zur Evaluation der optesVorkurse eingesetzt und ermöglicht inzwischen auch einen Vergleich der Ergebnisse über die Jahre und einzelnen Kohorten hinweg. Er ist auch Teil der Disseminationsmaterialien (siehe Kapitel 28).

\subsection{Evaluationsgegenstände und Evaluationsarten}

Die Arbeiten der einzelnen Teilprojekte im optes-Verbund führten zu verschiedenen Typen von (Zwischen-)Ergebnissen. Während zu Beginn der beiden Förderphasen vor allem theoretische Konzepte inklusive der Beschreibung notwendiger Rahmenbedingungen (z. B. Bedarfe für die Realisierung) entstanden sind, wurden mit fortschreitender Projektdauer auch konkrete Produkte (z. B. elektronische Lernmaterialien) fertiggestellt. Beide Typen von Projektergebnissen wurden formativ evaluiert, wobei sich der Fokus im Laufe des Projekts entsprechend von Konzept- zu Produkt- und Wirkungsevaluationen verschoben hat. In allen Phasen waren nicht nur einzelne Arbeitsergebnisse, sondern auch deren Kombination und Zusammenspiel von Interesse. Darüber hinaus wurden von Anfang an auch Prozesse bei der Evaluation mit einbezogen: zu Beginn primär Prozesse der Zusammenarbeit im Projektverbund, mit zunehmendem Projektfortschritt und entsprechend vorliegender optes-Angebote dann vor allem Prozesse, die mit der Implementation dieser Angebote an den Hochschulen zu tun haben. 


\subsection{Evaluationsbereiche und Evaluationsziele}

Neben der Unterscheidung der Evaluationsgegenstände in Konzepte, Produkte und Prozesse diente als weitere Ordnung für die geplanten Evaluationsmaßnahmen die Differenzierung von zwei unterschiedlichen Evaluationsbereichen, nämlich: Evaluationsmaßnahmen und -ziele, die sich auf die optes-Umgebung als solche beziehen, sowie Evaluationsmaßnahmen und -ziele, welche die optes-Implementation betreffen. Diese Einteilung erleichterte es, Vorgehensweisen in der Evaluation besser zu beschreiben sowie am Ende der Projektlaufzeit die Ergebnisse zueinander in Beziehung zu setzen und mit Blick auf das Gesamtprojekt zu interpretieren.

\subsubsection{Evaluationen der optes-Umgebung}

In formativen Evaluationen der optes-Umgebung wurden vor allem die Funktionsfähigkeit einzelner optes-Komponenten (damit sind sowohl materielle Ressourcen, wie einzelne Übungsaufgaben oder Lerntexte, als auch soziale Ressourcen, wie andere Lernende oder Lernbegleitende gemeint) sowie deren Wirkungen und Wechselwirkungen untersucht. Wie Studierende mit diesen Komponenten interagieren, hängt von verschiedenen Faktoren ab, die in der Person des Lernenden oder im Lernumfeld liegen. Zu den personenbezogenen Faktoren zählen beispielsweise persönliche Einstellungen und Wahrnehmungen, fachliche und überfachliche Fertigkeiten oder die Lernmotivation. Faktoren aus dem Lernumfeld können etwa die Unterstützung durch das persönliche (Arbeits-)Umfeld (z. B. die zur Verfügung stehende Zeit zum Lernen) oder die Qualität der Lernmaterialien sein. Maßnahmen in diesem Evaluationsbereich orientierten sich entsprechend an der Leitfrage, ob und wie gut aus den Interaktionen der Studierenden mit der optes-Umgebung, bzw. einzelner ihrer Komponenten, Lernsituationen entstehen, die einen erfolgreichen Lernprozess fördern. Aus der fortlaufenden Beantwortung dieser Frage wurden Maßnahmen zur Optimierung der optes-Angebote unter den gegebenen Möglichkeiten für die jeweilige Zielgruppe abgeleitet.

\subsubsection{Evaluationen der optes-Implementation}

Bei der formativen Evaluation von optes-Implementationen wurde zunächst danach unterschieden, ob es primär darum geht, (a) optes-Komponenten zu komplexen Lernumgebungen zu kombinieren und allgemein zur Nutzung anzubieten (z. B. die Kombination eines LoKs mit der Fähigkeitsmatrix und/oder dem Angebot zur Lernbegleitung), oder darum, (b) optes-Komponenten an einzelnen Hochschulen umzusetzen. Im ersten Fall richtete sich die Leitfrage auf die Kombinationsfähigkeit und das mögliche Zusammenspiel einzelner optes-Komponenten. Den Evaluationsergebnissen entsprechend reichte das Optimierungspotenzial von der 
Verbesserung einzelner Instruktionen für Lernende bis hin zur optimierten Verknüpfung ausgewählter optes-Komponenten. Im zweiten Fall waren zwei Leitfragen für die Evaluations handlungsrelevant:

1. Wie lassen sich die optes-Komponenten in Konzepte und Angebote einzelner Lehrender (Handlungsspielraum der Lehrenden) integrieren?

2. Wie lassen sich optes-Komponenten in die Studiengänge sowie in das Gesamtangebot der jeweiligen Hochschulen (Handlungsspielraum der Hochschule) integrieren?

Mit Blick auf Rollout und Verstetigung war es zudem wichtig zu erfahren, welche Szenarien der Implementierung von optes-Angeboten es bereits gibt und welche organisatorischen, finanziellen, personellen und konzeptionellen Rahmenbedingungen jeweils vorliegen. Das Erkenntnisinteresse lag beispielsweise auf der Erhebung notwendiger Rahmenbedingungen (z. B. hochschulspezifische Ressourcen), um bestimmte optes-Angebote an einer Einrichtung einsetzen zu können.

\subsection{Evaluationskriterien}

Die Evaluationskriterien für formative Evaluationsvorhaben im Rahmen des optesProjekts wurden, unabhängig vom jeweiligen Evaluationsbereich, grob in Kriterien zur Qualitätsbewertung und zur Wirkungsanalyse unterteilt. Kriterien zur Qualitätsbewertung dienen der Evaluation von Lernmaterialien und Konzepten. Sie beziehen sich auf didaktisch begründbare Qualitätsmerkmale einzelner optesKomponenten wie auch deren Integration und Implementation. Kriterien zur Wirkungsanalyse dienen der Evaluation von Nutzerinteraktionen mit der Lernumgebung. Diese umfassen nicht nur kognitive und organisationale Aspekte, sondern auch motivationale und emotionale Aspekte. Eine Liste mit Beispielen für die Evaluationskriterien sowie deren Zuordnung zu den Evaluationsbereichen findet sich nachfolgend in Tabelle 1.

Im Evaluationsbereich optes-Umgebung standen hingegen einzelne Lehr-LernInteraktionen und Lernsituationen im Fokus. Die Qualität von Lernmaterialien (um ein Beispiel zu nennen) kann etwa dahingehend bewertet werden, wie die LoKs im Einzelnen gestaltet sind, oder wie zweckmäßig das Angebot für das eigene Studium ist. Wirkungsanalysen können ein breites Spektrum annehmen: Evaluiert werden kann beispielweise, welchen Einfluss das Interesse der Lernenden an mathematischen Inhalten auf den Lernerfolg hat, wie hoch die Akzeptanz einzelner optes-Angebote ist oder wie die Studierenden den Zeit-Nutzen-Aufwand und ihren individuellen Lernzuwachs einschätzen. 
Tabelle 1: Kategorisierung der Evaluationskriterien für die jeweiligen Evaluationsbereiche anhand ausgewählter Beispiele

\begin{tabular}{|c|c|c|c|}
\hline & \multirow{2}{*}{$\begin{array}{c}\text { Kriterien zur } \\
\text { Qualitätsbewertung }\end{array}$} & \multicolumn{2}{|c|}{ Kriterien zur Wirkungsanalyse } \\
\hline & & $\begin{array}{l}\text { kognitiv und } \\
\text { organisational }\end{array}$ & $\begin{array}{l}\text { motivational und } \\
\text { emotional }\end{array}$ \\
\hline optes-Umgebung & $\begin{array}{l}\text { Gestaltung, Zweckmäßig- } \\
\text { keit fürs Studium, ... }\end{array}$ & $\begin{array}{l}\text { Lernzuwachs, Zeit- } \\
\text { Nutzen-Aufwand, ... }\end{array}$ & $\begin{array}{l}\text { Interesse, Akzep- } \\
\operatorname{tanz}, \ldots\end{array}$ \\
\hline $\begin{array}{l}\text { optes-Implemen- } \\
\text { tation }\end{array}$ & $\begin{array}{l}\text { Zweckmäßigkeit der } \\
\text { Kombination einzelner } \\
\text { Elemente, Passung zu den } \\
\text { Modulbeschreibungen, } \\
\text { Verbindlichkeit der In- } \\
\text { tegration, ... }\end{array}$ & $\begin{array}{l}\text { Auswirkung von Be- } \\
\text { treuung auf die Lern- } \\
\text { zeit, Ressourcenein- } \\
\text { satz der Hochschule } \\
\text {... }\end{array}$ & $\begin{array}{l}\text { Auswirkung von Be- } \\
\text { treuung auf die Mo- } \\
\text { tivation, empfundene } \\
\text { Arbeitserleichterung } \\
\text { beim Dozierenden, } \\
\ldots\end{array}$ \\
\hline
\end{tabular}

Im Evaluationsbereich optes-Implementation lag der Fokus auf Wechselbeziehungen einzelner Komponenten und der Integration in bestehende Handlungsspielräume von Lehrenden und Hochschulen. Die Qualität der Hochschul-Integration (um zwei Beispiele zu nennen) kann etwa dahingehend bewertet werden, wie gut sich eine Kombination einzelner Komponenten zur Erreichung eines bestimmten Zwecks eignet (z. B. die Kombination von Tests und Fähigkeitsmatrix zur persönlichen Lernstandskontrolle) oder wie gut das Angebot zu den Modulbeschreibungen passt und wie verbindlich das optes-Angebot in Studienprogramme aufgenommen wird. Wirkungsanalysen können sich auf zahlreiche Fragen beziehen: Evaluiert werden kann etwa, wie sich die Betreuung der Studierenden durch Lernbegleitende bei der Bearbeitung der LoKs auf die Motivation und die Lernzeit auswirkt oder welchen Einfluss vorhandene Ressourcen an der Hochschule auf die Arbeitserleichterung für Lehrende bei der optes-Nutzung haben.

\subsection{Evaluationsmethoden}

Je nach Projektphase, Evaluationsgegenstand, Erkenntnisinteresse und Fragestellung wurden für die formative Evaluation verschiedene Evaluationsmethoden eingesetzt: Befragungen, Beobachtungen und Dokumentanalysen.

In den Konzept- und Entwicklungsphasen boten sich beispielsweise Dokumentanalysen an, um Ansatzpunkte für die Umsetzung neuer Themenschwerpunkte, wie die Förderung des reflektierten Übens, oder für Weiterentwicklungen in den bestehenden optes-Konzepten zu identifizieren.

In den Pilotphasen ließen sich vor allem die Wirkungen der bis dahin erarbeiteten und implementierten Maßnahmen evaluieren. (Online-)Befragungen der Nutzer*innen waren hier eine ökonomische Methode, um möglichst viele quantitative Daten zu generieren, auf deren Basis sich statistisch belastbare Aussagen über die 
Wirkung des optes-Angebots treffen lassen. Aufgrund der unterschiedlichen Implementierung verschiedener optes-Komponenten an den unterschiedlichen Standorten (beispielsweise wurden die Online-Lernangebote an verschiedenen Standorten variabel mit den dort existierenden Präsenzangeboten verwoben) ließen sich die so erhobenen Daten zwar nur bedingt direkt miteinander vergleichen, erlaubten aber kontextualisierte Schlüsse hinsichtlich der Wirksamkeit innerhalb eines Standortes.

Schließlich spielt im Rahmen des optes-Projekts die Usability der elektronischen Lernumgebung eine zentrale Rolle. Um diese zu ergründen, bot es sich an, die Lernenden bei der konkreten Nutzung des Angebots zu beobachten und so Erkenntnisse über Navigation, Nutzerfreundlichkeit und weitere Usability-Aspekte zu erhalten.

\subsection{Rückmeldungen an das Projekt}

Damit die Ergebnisse aus formativen Evaluationen wirksam sein und identifizierte Optimierungsbedarfe im Sinne eines zyklischen Re-Designs auch umgesetzt werden konnten, mussten diese als Rückmeldungen in das Projekt zurückfließen. Diese Rückmeldungen sind auf unterschiedlichen Wegen erfolgt.

Alle entstandenen Konzepte wurden den Projektpartnern zur Verfügung gestellt und im Gesamtprojekt diskutiert. Das galt beispielsweise für den Theorierahmen zum reflektierten Üben, für das didaktische Beratungskonzept, für theoretische Ausarbeitungen zur Motivation oder auch die Evaluationskonzepte.

Außerdem wurden die Partner durch Ergebnisberichte über die Erkenntnisse aus den durchgeführten Evaluationen informiert. Teilprojekte, die von den identifizierten Optimierungsbedarfen direkt betroffen waren, wurden von der wissenschaftlichen Begleitung darüber hinaus in einem Beratungsgespräch, bei dem auch Verbesserungsvorschläge sowie deren Umsetzung diskutiert werden konnten, informiert.

Erkenntnisse aus den Arbeitsgruppen flossen in Form von Zwischen- und Abschlussberichten in das Gesamtprojekt zurück. In diesen wurden die bisherigen Erkenntnisse sowie die weiteren Schritte dokumentiert und so für die künftige Projektarbeit zugänglich gemacht.

\subsection{Abschließende Erkenntnisse}

Während der gesamten Projektlaufzeit wurden bisher insgesamt 29 formative Evaluationsmaßnahmen - entweder von der wissenschaftlichen Begleitung oder gerade zu Beginn des Projekts von den Teilprojekten selbst - durchgeführt, mehrere Kohorten von Studienanfänger*innen und zahlreiche Lehrende befragt. Die Er- 
kenntnisse, die aus diesen Maßnahmen gewonnen wurden, haben dazu beigetragen, die optes-Angebote sukzessive zu optimieren und an die Bedürfnisse der Zielgruppen anzupassen.

Die letzten Evaluationsergebnisse weisen darauf hin, dass die im Verbundprojekt erarbeiteten Angebote und Produkte von den Nutzer*innen positiv wahrgenommen werden. Nach anfänglichen Schwierigkeiten hat sich vor allem die Beurteilung der Usability und der Qualität der Materialien im Laufe des Projekts zunehmend verbessert. Die positive Wahrnehmung zeigt sich auch darin, dass viele Befragte die optes-Angebote und die umfangreichen Lehr-Lern-Materialien weiterempfehlen würden. Allerdings deuten die in fast allen Nutzerbefragungen auffallend hohen Standardabweichungen darauf hin, dass es einige Nutzer*innen gibt, die mit den Angeboten deutlich besser zurechtkommen und diese positiver bewerten als andere. Worauf sich dies zurückführen lässt, ist mit den verfügbaren Daten leider nicht zu beantworten.

Abschließend lässt sich festhalten, dass sich der im optes-Projekt verfolgte Evaluationsansatz und dessen phasenspezifische Anpassung weitgehend bewährt haben. Auch wenn der Zugang zu den Zielgruppen nicht immer einfach war, gelang es durch Anreize, wie beispielsweise Druckguthaben für Studierende, oder durch persönliche Ansprache akzeptable Rücklaufquoten zu erzielen. Die in der formativen Evaluation gewonnenen Erkenntnisse haben letztlich in hohem Maße zur Qualität der Projektergebnisse beigetragen.

\section{Literatur}

Patton, M. Q. (2010). Developmental Evaluation. Applying complexity concepts to enhance innovation and use. New York: Guilford Press.

Reinmann, G. (2004). Qualitätsmanagement in Schulen: Eine Einführung. In R. Arnold \& C. Griese (Hrsg.), Schulleitung und Schulentwicklung (S. 45-165). Hohengehren: Schneider Verlag.

Scriven, M. (1991). Evaluation Theasaurus. Newbury Park, CA: Sage.

Wottawa, H. (2006). Evaluation. In A. Krapp \& B. Weidenmann (Hrsg.), Pädaggische Psychologie (S. 659-687). Weinheim: Beltz. 
Open Access Dieses Kapitel wird unter der Creative Commons Namensnennung 4.0 International Lizenz (http://creativecommons.org/licenses/by/4.0/deed. de) veröffentlicht, welche die Nutzung, Vervielfältigung, Bearbeitung, Verbreitung und Wiedergabe in jeglichem Medium und Format erlaubt, sofern Sie den/ die ursprünglichen Autor(en) und die Quelle ordnungsgemäß nennen, einen Link zur Creative Commons Lizenz beifügen und angeben, ob Änderungen vorgenommen wurden.

Die in diesem Kapitel enthaltenen Bilder und sonstiges Drittmaterial unterliegen ebenfalls der genannten Creative Commons Lizenz, sofern sich aus der Abbildungslegende nichts anderes ergibt. Sofern das betreffende Material nicht unter der genannten Creative Commons Lizenz steht und die betreffende Handlung nicht nach gesetzlichen Vorschriften erlaubt ist, ist für die oben aufgeführten Weiterverwendungen des Materials die Einwilligung des jeweiligen Rechteinhabers einzuholen.

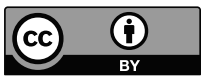

